Commun. Korean Math. Soc. 25 (2010), No. 3, pp. 349-364

DOI 10.4134/CKMS.2010.25.3.349

\title{
RING ENDOMORPHISMS WITH THE REVERSIBLE CONDITION
}

\author{
Muhittin Başer, Fatma Kaynarca, and Tai Keun KwaK
}

\begin{abstract}
P. M. Cohn called a ring $R$ reversible if whenever $a b=0$, then $b a=0$ for $a, b \in R$. Commutative rings and reduced rings are reversible. In this paper, we extend the reversible condition of a ring as follows: Let $R$ be a ring and $\alpha$ an endomorphism of $R$, we say that $R$ is right (resp., left) $\alpha$-shifting if whenever $a \alpha(b)=0$ (resp., $\alpha(a) b=0$ ) for $a, b \in R$, $b \alpha(a)=0$ (resp., $\alpha(b) a=0$ ); and the ring $R$ is called $\alpha$-shifting if it is both left and right $\alpha$-shifting. We investigate characterizations of $\alpha$ shifting rings and their related properties, including the trivial extension, Jordan extension and Dorroh extension. In particular, it is shown that for an automorphism $\alpha$ of a ring $R, R$ is right (resp., left) $\alpha$-shifting if and only if $Q(R)$ is right (resp., left) $\bar{\alpha}$-shifting, whenever there exists the classical right quotient ring $Q(R)$ of $R$.
\end{abstract}

\section{Introduction}

Throughout this paper all rings are associative with identity, and we assume that every endomorphism of a ring is a nonzero and non identity endomorphism, unless specified otherwise. Recall that a ring is reduced if it has no nonzero nilpotent elements, Cohn [5] called a ring $R$ reversible if $a b=0$ implies $b a=0$ for $a, b \in R$; while Habeb [6] used the term reversible for what is called zero commutative. The class of reversible rings was extended to the class of rings satisfying $Z C_{n}$ for $n \geq 2$ by Anderson and Camillo [2]. A ring $R$ satisfies $Z C_{n}$ for $n \geq 2$ if for $a_{1}, \ldots, a_{n} \in R$ with $a_{1} \cdots a_{n}=0, a_{\sigma(1)} \cdots a_{\sigma(n)}=0$ for each $\sigma \in \Sigma_{n}$ where $\Sigma_{n}$ denotes the permutation group on $n$ letters. Every reduced ring satisfies $Z C_{n}$ for all $n \geq 2$ [2, Theorem I.3], but the converse does not hold [2, Example II.5]. Due to Narbonne [17], a ring $R$ is called semicommutative if $a b=0$ implies $a R b=0$ for $a, b \in R$. Some of the earliest results known to us about semicommutative rings (although not so called at the time) were due to Shin [21], in particular, it was shown that $R$ is a semicommutative ring if and only if for each $a \in R, r_{R}(a)$ is an ideal of $R$ where $r_{R}(a)=\{b \in R \mid a b=0\}$

Received September 12, 2009; Revised December 26, 2009.

2000 Mathematics Subject Classification. Primary 16W20, 16U80; Secondary 16S36.

Key words and phrases. ring endomorphism, reduced ring, reversible ring, trivial extension, classical right quotient ring. 
[21, Lemma 1.2]. Semicommutative rings were also studied under the name zero insertive by Habeb [6]. It is well-known that commutative rings or reduced rings are reversible, and reversible rings are semicommutative, but the converses do not hold, respectively.

Recently, the concept of the reversible condition of elements at zero is extended to one of both an endomorphism and an element in a ring. From [3, Definition 2.1], an endomorphism $\alpha$ of a ring $R$ is called right (resp., left) reversible if whenever $a b=0$ for $a, b \in R, b \alpha(a)=0$ (resp., $\alpha(b) a=0$ ). A ring $R$ is called right (resp., left) $\alpha$-reversible if there exists a right (resp., left) reversible endomorphism $\alpha$ of $R$. The ring $R$ is $\alpha$-reversible if it is both right and left $\alpha$-reversible. It is natural to consider the following condition $(*)$ for an endomorphism $\alpha$ of a ring $R$,

(*) $\quad a \alpha(b)=0$ for $a, b \in R$ implies $b \alpha(a)=0$.

The $\alpha$-reversible condition and the condition (*) above of a ring do not dependent on each other by the next example.

Example 1.1. (1) Let $\mathbb{Z}_{2}$ be the ring of integers modulo 2 and consider the $\operatorname{ring} R=\mathbb{Z}_{2} \oplus \mathbb{Z}_{2}$ with the usual addition and multiplication. Let $\alpha: R \rightarrow R$ be defined by $\alpha((a, b))=(b, a)$. For $(a, b),(c, d) \in R,(a, b) \alpha((c, d))=0 \Leftrightarrow a d=0$ and $b c=0 \Leftrightarrow(c, d) \alpha((a, b))=0$, concluding that $R$ satisfies the condition $(*)$. Note that $R$ is neither right nor left $\alpha$-reversible by [3, Example 2.3].

(2) Let $R=F[x]$ be the polynomial ring over a field $F$ and $\alpha: R \rightarrow R$ defined by $\alpha(f(x))=f(0)$ where $f(x) \in R$. Then $R$ is a commutative domain (and so reduced) and $\alpha$ is not a monomorphism. By [3, Example 2.7(ii)], $R$ is right $\alpha$-reversible. However, $R$ does not satisfy the condition $(*)$ : In fact, for $f(x)=a \neq 0$ and $g(x)=x \in R$ we have $f(x) \alpha(g(x))=0$, but $g(x) \alpha(f(x)) \neq 0$.

Recall that an endomorphism $\alpha$ of a ring $R$ is called rigid [13] if $a \alpha(a)=0$ implies $a=0$ for $a \in R$, and $R$ is called an $\alpha$-rigid ring [7] if there exists a rigid endomorphism $\alpha$ of $R$. Rigid endomorphisms are monomorphisms, and $\alpha$-rigid rings are reduced by [7, Proposition 5]. Moreover, every $\alpha$-rigid ring is $\alpha$-reversible, but the converse does not hold by [3, Proposition 2.5 and Example 2.7]. As a parallel result to this, we have the following for a ring satisfying the condition $(*)$.

Proposition 1.2. For an endomorphism $\alpha$ of a ring $R$, the following are equivalent:

(1) $R$ is an $\alpha$-rigid ring.

(2) $R$ satisfies the condition $(*)$ and $a R \alpha(a)=0$ implies $a=0$ for any $a \in R$.

Proof. Assume that $R$ is an $\alpha$-rigid ring. It is clear that $a R \alpha(a)=0$ implies $a=0$ for $a \in R$. Let $a \alpha(b)=0$ for any $a, b \in R$. Then $b a \alpha(b a)=b a \alpha(b) \alpha(a)=$ $0 \Rightarrow b a=0 \Rightarrow a b=0 \Rightarrow b \alpha(a) \alpha(b \alpha(a))=b \alpha(a b) \alpha^{2}(a)=0 \Rightarrow b \alpha(a)=0$, since $R$ is $\alpha$-rigid and so reduced. Therefore $R$ satisfies (*). Conversely, assume (2). 
Let $a \alpha(a)=0$ for $a \in R$. For any $r \in R, 0=a \alpha(a) \alpha(r)=a \alpha(a r)$ implies $\operatorname{ar} \alpha(a)=0$ and hence, $a=0$ by assumption. Thus $R$ is $\alpha$-rigid.

Corollary 1.3 ([12, Lemma 2.7]). A ring $R$ is reduced if and only if $R$ is a semiprime and reversible ring.

Proof. It follows from Proposition 1.2, letting $\alpha=i d_{R}$ where $i d_{R}$ denotes the identity endomorphism of a ring $R$.

The condition " $a R \alpha(a)=0$ implies $a=0$ for any $a \in R$ " in Proposition 1.2(2) cannot be dropped: For the ring $R=\mathbb{Z}_{2} \oplus \mathbb{Z}_{2}$ with an automorphism $\alpha$ in Example 1.1(1), the ring $R$ is not $\alpha$-rigid and for $0 \neq a=(1,0) \in R, a R \alpha(a)=$ 0 . Hence, the class of rings satisfying the condition $(*)$ is a natural extension of reversible rings as well as a generalization of $\alpha$-rigid rings, independently from the class of $\alpha$-reversible rings (in [3]).

\section{2. $\alpha$-Shifting rings and their properties}

Based on the arguments above, we define the following.

Definition 2.1. Let $R$ be a ring and $\alpha$ an endomorphism of $R$, we say that $R$ is right (resp., left) $\alpha$-shifting if whenever $a \alpha(b)=0$ (resp., $\alpha(a) b=0$ ) for $a, b \in R, b \alpha(a)=0$ (resp., $\alpha(b) a=0$ ); and the $\operatorname{ring} R$ is called $\alpha$-shifting if it is both left and right $\alpha$-shifting.

Remark 2.2. (1) A ring $R$ is reversible if $R$ is one-sided $i d_{R}$-shifting, where $i d_{R}$ is the identity endomorphism of $R$. Every subring $S$ with $\alpha(S) \subseteq S$ of a right (resp., left) $\alpha$-shifting ring is also right (resp., left) $\alpha$-shifting ring.

(2) Let $R_{\gamma}$ be a ring and $\alpha_{\gamma}$ an endomorphism of $R_{\gamma}$ for each $\gamma \in \Gamma$. Then, for the product $\prod_{\gamma \in \Gamma} R_{\gamma}$ of $R_{\gamma}$ and the endomorphism $\alpha: \prod_{\gamma \in \Gamma} R_{\gamma} \rightarrow \prod_{\gamma \in \Gamma} R_{\gamma}$ defined by $\alpha\left(\left(a_{\gamma}\right)_{\gamma \in \Gamma}\right)=\left(\alpha_{\gamma}\left(a_{\gamma}\right)\right)_{\gamma \in \Gamma}, \prod_{\gamma \in \Gamma} R_{\gamma}$ is right (resp., left) $\alpha$-shifting if and only if $R_{\gamma}$ is right (resp., left) $\alpha_{\gamma}$-shifting for each $\gamma \in \Gamma$.

Observe that Example 1.1(2) illuminates that there exists a commutative domain which is not $\alpha$-shifting where $\alpha$ is not a monomorphism. However, it can be easily checked that any domain $R$ with a monomorphism $\alpha$ is both right and left $\alpha$-shifting; but the converse does not hold by the following example which also shows that the concepts of reduced rings and $\alpha$-shifting rings do not dependent on each other with Example 1.1(2).

Example 2.3. For the ring $\mathbb{Z}_{4}$ of integers modulo 4 , consider the ring $R=$ $\left\{\left(\begin{array}{ll}a & b \\ 0 & a\end{array}\right) \mid a, b \in \mathbb{Z}_{4}\right\}$ and an endomorphism $\alpha$ defined by $\alpha\left(\left(\begin{array}{ll}a & b \\ 0 & a\end{array}\right)\right)=\left(\begin{array}{cc}a & -b \\ 0 & a\end{array}\right)$. Then $R$ is commutative (and hence reversible), but not reduced. Hence, $R$ is $\alpha$-shifting by Theorem 2.6(2)(below).

For a nonempty subset $X$ of a ring $R$, we write $r_{R}(X)=\{c \in R \mid X c=0\}$ which is called the right annihilator of $X$ in $R$. Similarly, $\ell_{R}(X)$ denotes the left annihilator of $X$ in $R$. 
Proposition 2.4. Let $R$ be a ring with an endomorphism $\alpha$. The following are equivalent:

(1) $R$ is a right (resp., left) $\alpha$-shifting ring.

(2) $\alpha\left(\ell_{R}(\alpha(B))\right) \subseteq r_{R}(B)\left(\right.$ resp., $\left.\alpha\left(r_{R}(\alpha(B))\right) \subseteq \ell_{R}(B)\right)$ for any nonempty subset $B$ of $R$.

(3) $\alpha\left(\ell_{R}(\alpha(b))\right) \subseteq r_{R}(b)\left(\right.$ resp., $\left.\alpha\left(r_{R}(\alpha(b))\right) \subseteq \ell_{R}(b)\right)$ for any $b \in R$.

(4) $A \alpha(B)=0 \Leftrightarrow B \alpha(A)=0$ (resp., $\alpha(A) B=0 \Leftrightarrow \alpha(B) A=0$ ) for any nonempty subsets $A, B$ of $R$.

Proof. (1) $\Rightarrow(2)$ Assume that $R$ is right $\alpha$-shifting. Let $a \in \alpha\left(\ell_{R}(\alpha(B))\right)$. Then $a=\alpha(c)$ and $c \alpha(B)=0$ for some $c \in R$. Then for any $b \in B, c \alpha(b)=0$ and so $b \alpha(c)=b a=0$ by assumption. Hence, $a \in r_{R}(B)$, concluding that $\alpha\left(\ell_{R}(\alpha(B))\right) \subseteq r_{R}(B) . \quad(2) \Rightarrow(3)$ and $(4) \Rightarrow(1)$ are obvious. (3) $\Rightarrow(4)$ Assume (3). Let $A \alpha(B)=0$ for any nonempty subsets $A, B$ of $R$. Then $a \alpha(b)=0$ for $a \in A$ and $b \in B$. Since $a \in \ell_{R}(\alpha(b)), \alpha(a) \in r_{R}(b)$ by assumption, and so $b \alpha(a)=0$. Thus $B \alpha(A)=\sum_{a \in A, b \in B} b \alpha(a)=0$. Similarly, $B \alpha(A)=0$ implies $A \alpha(B)=0$. The proof for a left $\alpha$-shifting ring is similar to the above.

The next example shows that the $\alpha$-shiftability is not right-left symmetric; in addition, it shows that the converse of (3) in Proposition 2.4 does not hold.

Example 2.5. Consider a ring $R=\left\{\left(\begin{array}{ll}a & b \\ 0 & c\end{array}\right) \mid a, b, c \in \mathbb{Z}\right\}$, where $\mathbb{Z}$ is the ring of integers. For $A=\left(\begin{array}{ll}1 & 0 \\ 0 & 0\end{array}\right)$ and $B=\left(\begin{array}{ll}0 & 0 \\ 0 & 1\end{array}\right)$ in $R, A B=0$, but $0 \neq A\left(\begin{array}{ll}1 & 1 \\ 0 & 0\end{array}\right) B \in$ $A R B$. Thus $R$ is not semicommutative, and so it is not reversible.

(1) Let $\alpha: R \rightarrow R$ be an endomorphism defined by $\alpha\left(\left(\begin{array}{ll}a & b \\ 0 & c\end{array}\right)\right)=\left(\begin{array}{ll}a & 0 \\ 0 & 0\end{array}\right)$. Let $A=\left(\begin{array}{ll}a & b \\ 0 & c\end{array}\right)$ and $B=\left(\begin{array}{cc}a^{\prime} & b^{\prime} \\ 0 & c^{\prime}\end{array}\right) \in R$. Then $A \alpha(B)=0$ if and only if $a a^{\prime}=0$ if and only if $B \alpha(A)=0$, entailing that $R$ is right $\alpha$-shifting. However, for $A=\left(\begin{array}{ll}0 & 1 \\ 0 & 1\end{array}\right)$ and $B=\left(\begin{array}{ll}1 & 1 \\ 0 & 0\end{array}\right) \in R$ with $\alpha(A) B=0$, we have $\alpha(B) A \neq 0$, and thus $R$ is not left $\alpha$-shifting. Note that for $B=\left(\begin{array}{ll}1 & 1 \\ 0 & 0\end{array}\right) \in R, r_{R}(B)=\left\{\left(\begin{array}{cc}0 & b \\ 0 & -b\end{array}\right) \mid b \in \mathbb{Z}\right\}$ and $\alpha\left(\ell_{R}(\alpha(B))\right)=\left\{\left(\begin{array}{ll}0 & 0 \\ 0 & 0\end{array}\right)\right\}$, concluding that $r_{R}(B) \nsubseteq \alpha\left(\ell_{R}(\alpha(B))\right)$.

(2) Let $\beta: R \rightarrow R$ be an endomorphism defined by $\beta\left(\left(\begin{array}{ll}a & b \\ 0 & c\end{array}\right)\right)=\left(\begin{array}{ll}0 & 0 \\ 0 & c\end{array}\right)$. By the similar method to (1), it is shown that $R$ is left $\beta$-shifting, but not right $\beta$-shifting. Moreover, for $B=\left(\begin{array}{ll}0 & 1 \\ 0 & 1\end{array}\right) \in R, \ell_{R}(B)=\left\{\left(\begin{array}{cc}-b & b \\ 0 & 0\end{array}\right) \mid b \in \mathbb{Z}\right\} \nsubseteq$ $\beta\left(r_{R}(\beta(B))\right)=\left\{\left(\begin{array}{ll}0 & 0 \\ 0 & 0\end{array}\right)\right\}$.

Theorem 2.6. For a ring $R$ with an endomorphism $\alpha$, we have the following.

(1) Let $R$ be a reversible ring. Then $R$ is right $\alpha$-shifting if and only if $R$ is left $\alpha$-shifting.

(2) Let $\alpha^{2}=i d_{R}$ where $i d_{R}$ is the identity endomorphism of $R$. Then $R$ is right (or left) $\alpha$-shifting if and only if $R$ is reversible.

(3) Let $R$ be a right (resp., left) $\alpha$-shifting ring. Then the following are equivalent: (i) $\alpha$ is a monomorphism; (ii) $\alpha(1)=1$ where 1 is the identity of $R$; (iii) $a b=0 \Leftrightarrow b \alpha^{2}(a)=0$ (resp., $\left.\alpha^{2}(b) a=0\right)$.

(4) If $R$ is right (or left) $\alpha$-shifting and $\alpha$ is a monomorphism, then $R$ is semicommutative. 
(5) The following are equivalent: (i) $R$ is an $\alpha$-rigid ring; (ii) $R$ is a right $\alpha$-shifting ring and $a R \alpha(a)=0$ implies $a=0$ for any $a \in R$; (iii) $R$ is a left $\alpha$-shifting ring and $\alpha(a) R a=0$ implies $a=0$ for any $a \in R$.

(6) Let $R$ be a reversible ring and $\alpha$ is a monomorphism of $R$. If $R$ is $\alpha$-reversible, then $R$ is $\alpha$-shifting.

Proof. (1) Suppose that $R$ is right $\alpha$-shifting. For $a, b \in R, \alpha(a) b=0 \Rightarrow$ $b \alpha(a)=0 \Rightarrow a \alpha(b)=0 \Rightarrow \alpha(b) a=0$, since $R$ is right $\alpha$-shifting and reversible. Thus $R$ is left $\alpha$-shifting. The converse is similar.

(2) Suppose that $R$ is right $\alpha$-shifting. For $a, b \in R, a b=0 \Rightarrow \alpha(a) \alpha(b)=$ $0 \Rightarrow b \alpha^{2}(a)=0 \Rightarrow b a=0$. Thus $R$ is reversible. Conversely, assume that $R$ is reversible. For $a, b \in R, a \alpha(b)=0 \Rightarrow \alpha(b) a=0 \Rightarrow \alpha^{2}(b) \alpha(a)=0 \Rightarrow b \alpha(a)=0$, entailing that $R$ is right $\alpha$-shifting. By the similar arguments, it is proven that $R$ is left $\alpha$-shifting if and only if $R$ is reversible.

(3) (i) $\Leftrightarrow(i i)$ : Assume (i). Then $(1-\alpha(1)) \alpha(1)=0$ implies $\alpha(1-\alpha(1))=0$ and hence $\alpha(1)=1$ by hypothesis. Conversely, let $\alpha(1)=1$. If $\alpha(a)=\alpha(b)$ for $a, b \in R$, then $\alpha(a-b)=0 \Rightarrow(a-b) \alpha(1)=0 \Rightarrow a=b$, concluding that $\alpha$ is a monomorphism. (i) $\Leftrightarrow($ iii): Assume (i). For $a, b \in R, a b=0 \Leftrightarrow \alpha(a) \alpha(b)=$ $0 \Leftrightarrow b \alpha^{2}(a)=0$. Assume (iii). If $\alpha(a)=\alpha(b)$ for $a, b \in R$, then $\alpha^{2}(a-b)=0$ and so $a-b=0$, entailing that $\alpha$ is a monomorphism.

(4) Suppose that $R$ is right $\alpha$-shifting and $\alpha$ is a monomorphism. For $a, b \in R$ $a b=0 \Rightarrow \alpha(a) \alpha(b)=0 \Rightarrow b \alpha(\alpha(a))=0$, and hence for any $r \in R, r b \alpha(\alpha(a))=$ $0 \Rightarrow \alpha(a) \alpha(r b)=0 \Rightarrow \alpha(a r b)=0 \Rightarrow a r b=0$ by hypothesis. Similarly, we have the same conclusion for a left $\alpha$-shifting ring.

(5) (i) $\Leftrightarrow$ (ii) follows from Proposition 1.2. (i) $\Rightarrow$ (iii) follows from (ii) and (1), since every $\alpha$-rigid ring is reduced by [7, Proposition 5]. (iii) $\Rightarrow$ (i): Assume (iii). It is enough to show that $\alpha(a) a=0$ implies $a=0$ for $a \in R$ by $[9$, Proposition 2.4]. Let $\alpha(a) a=0$ for $a \in R$. Then for any $r \in R$, we have $0=\alpha(r) \alpha(a) a=\alpha(r a) a$, and so $\alpha(a) r a=0$. Hence $\alpha(a) R a=0$ and so $a=0$, proving that $R$ is $\alpha$-rigid.

(6) Assume that $R$ is $\alpha$-reversible. Let $a \alpha(b)=0 \Rightarrow \alpha(a) \alpha(b)=0 \Rightarrow$ $\alpha(b) \alpha^{2}(a)=0 \Rightarrow b \alpha(a)=0$, completing the proof.

Corollary 2.7 ([14, Proposition 1.3] and [12, Lemma 1.4]). Reversible rings are semicommutative.

Proof. It follows directly from Theorem 2.6(4), letting $\alpha=i d_{R}$.

Notice that Example 1.1(2) and Example 2.5(1) show that the class of reversible rings is independent of the class of right $\alpha$-shifting rings. In relating to Theorem 2.6(3), we may ask that $\alpha(1)=1$ implies $\alpha(e)=e$ for any $e^{2}=e \in R$, but the possibility is erased by Example 1.1(1) which also shows that the converse of Theorem 2.6(6) does not hold, and by the results in Theorem 2.6(3), it can be useful to observe that any ring with a unitary endomorphism $\alpha$ which is not a monomorphism, can not be left nor right $\alpha$-shifting; and the condition " $\alpha$ is a monomorphism" in Theorem 2.6(4), cannot be dropped by Example 
2.5(1) and there exists a semicommutative ring with an endomorphism $\alpha$ which is neither right nor left $\alpha$-shifting by the next example.

Example 2.8. Let $R=\left\{\left(\begin{array}{ll}a & b \\ 0 & a\end{array}\right) \mid a, b \in \mathbb{Z}\right\}$ and $\alpha$ be defined by $\alpha\left(\left(\begin{array}{ll}a & b \\ 0 & a\end{array}\right)\right)=$ $\left(\begin{array}{ll}a & 0 \\ 0 & a\end{array}\right)$. Then $R$ is commutative (and so semicommutative). For $A=\left(\begin{array}{ll}1 & 0 \\ 0 & 1\end{array}\right)$ and $B=\left(\begin{array}{ll}0 & 1 \\ 0 & 0\end{array}\right) \in R, A \alpha(B)=0$ but $B \alpha(A) \neq 0$. Thus $R$ is not right $\alpha$-shifting. Similarly it can be shown that $R$ is not left $\alpha$-shifting, either.

Recall that for an endomorphism $\alpha$ of a ring $R$, the skew polynomial ring $R[x ; \alpha]$ of $R$ consists of the polynomial in $x$ with coefficients in $R$ written on the left, subject to the relation $x r=\alpha(r) x$ for all $r \in R$.

Theorem 2.9. Let $R$ be a ring with an endomorphism $\alpha$.

(1) If $R$ is an abelian ring (i.e., all its idempotents are central) with $\alpha(e)=e$ for any $e^{2}=e \in R$, then every idempotent of $R[x ; \alpha]$ is in $R$ and $R[x ; \alpha]$ is abelian.

(2) If $R$ is a right (resp., left) $\alpha$-shifting ring with $\alpha(e)=e$ for any $e^{2}=e \in$ $R$, then both $R$ and $R[x ; \alpha]$ are abelian.

(3) If $R[x ; \alpha]$ is a reversible ring, then $R$ is an $\alpha$-shifting ring.

Proof. (1) Assume that $R$ is abelian with $\alpha(e)=e$ for any $e^{2}=e \in R$. Let $f^{2}=f \in R[x ; \alpha]$, where $f=e_{0}+e_{1} x+e_{2} x^{2}+\cdots+e_{n} x^{n}$. Then

$$
\sum_{k=0}^{n}\left(\sum_{i+j=k} e_{i} \alpha^{i}\left(e_{j}\right)\right) x^{k}=\sum_{k=0}^{n} e_{k} x^{k} .
$$

When $k=0$, we get $e_{0}^{2}=e_{0}$, and so $e_{0}$ is central and $\alpha\left(e_{0}\right)=e_{0}$ by assumption. For the coefficient of $x$ in Eq.(1), $e_{0} e_{1}+e_{1} \alpha\left(e_{0}\right)=e_{1}$ implies $2 e_{1} e_{0}=e_{1} \Rightarrow$ $2 e_{1} e_{0}\left(1-e_{0}\right)=e_{1}\left(1-e_{0}\right) \Rightarrow e_{1}=e_{1} e_{0} \Rightarrow e_{1}=0$. From the coefficient of $x^{2}$ in Eq.(1), $e_{0} e_{2}+e_{2} e_{0}=e_{2}$ yields $2 e_{0} e_{2}=e_{2}$ and so $e_{2}=0$ by the same method as above. Continuing this procedure implies $e_{i}=0$ for $i \geq 1$. Consequently, $f=e_{0}=e_{0}^{2} \in R$ and hence the set of all idempotents in $R[x ; \alpha]$ coincides with the set of all idempotents of $R$, entailing that $R[x ; \alpha]$ is abelian.

(2) It is enough to show that $R$ is abelian by (1). Assume that $R$ is a right $\alpha$-shifting ring with $\alpha(e)=e$ for any $e^{2}=e \in R$. Let $e^{2}=e \in R$. Then $e(1-e)=0$ and $(1-e) e=0$. By assumption, we get $e \alpha(1-e)=0$ and $(1-e) \alpha(e)=0$. For any $r \in R, e \alpha(1-e) \alpha(r)=0$ and $(1-e) \alpha(e) \alpha(r)=0 \Rightarrow$ $0=(1-e) r \alpha(e)=(1-e) r e$ and $0=e r \alpha(1-e)=e r(1-e)$, proving that $R$ is an abelian ring.

(3) Suppose that $R[x ; \alpha]$ is reversible. Let $a \alpha(b)=0$ for any $a, b \in R$. For $p(x)=a x, q(x)=b x \in R[x ; \alpha], p(x) q(x)=a \alpha(b) x^{2}=0$, and hence $q(x) p(x)=b \alpha(a) x^{2}=0$, namely $b \alpha(a)=0$. Thus $R$ is right $\alpha$-shifting. Since $R$ is reversible as a subring of $R[x ; \alpha], R$ is $\alpha$-shifting by Theorem 2.6(1).

In the following example, the part (1) illuminates that the condition " $\alpha(e)=$ $e$ for any $e^{2}=e \in R$ " of Theorem 2.9(1) and (2) cannot be dropped respectively; and the part (2) shows that the converse of Theorem 2.9(3) does not 
hold; and the part (3) shows that the condition " $R[x ; \alpha]$ is a reversible ring" in Theorem 2.9(3) cannot be replaced by " $R[x ; \alpha]$ is a semicommutative ring".

Example 2.10. (1) The ring $R=\mathbb{Z}_{2} \oplus \mathbb{Z}_{2}$ with $\alpha$ defined by $\alpha((a, b))=(b, a)$ in Example 1.1(1) is abelian and right $\alpha$-shifting, but $\alpha((1,0)) \neq(1,0)$ for an idempotent $(1,0)$ of $R$. Moreover, $p(x)=(1,0)+(0,1) x$ is an idempotent of $R[x ; \alpha]$, but $p(x) \notin R$ and $p(x) q(x) \neq q(x) p(x)$ for $q(x)=(1,0) x \in R[x ; \alpha]$.

(2) In Example 2.5(1), the ring $R=\left\{\left(\begin{array}{ll}a & b \\ 0 & c\end{array}\right) \mid a, b, c \in \mathbb{Z}\right\}$ and $\alpha$ defined by $\alpha\left(\left(\begin{array}{ll}a & b \\ 0 & c\end{array}\right)\right)=\left(\begin{array}{ll}a & 0 \\ 0 & 0\end{array}\right)$ is right $\alpha$-shifting. For $p(x)=\left(\begin{array}{ll}0 & 1 \\ 0 & 0\end{array}\right)+\left(\begin{array}{ll}0 & 1 \\ 0 & 0\end{array}\right) x, q(x)=\left(\begin{array}{ll}1 & 1 \\ 0 & 0\end{array}\right) \in$ $R[x ; \alpha]$, we have $p(x) q(x)=0$. But $q(x) p(x)=\left(\begin{array}{cc}0 & 1 \\ 0 & 0\end{array}\right)+\left(\begin{array}{cc}0 & 1 \\ 0 & 0\end{array}\right) x \neq 0$, so $R[x ; \alpha]$ is not reversible.

(3) The commutative domain $R=F[x]$ with $\alpha$ defined by $\alpha(f(x))=f(0)$ for $f(x) \in R$ in Example 1.1(2) is not $\alpha$-shifting. We claim that $R[y ; \alpha]$ is a semicommutative ring. Let $p(y)=f_{0}(x)+f_{1}(x) y+\cdots+f_{m}(x) y^{m}$ and $q(y)=$ $g_{0}(x)+g_{1}(x) y+\cdots+g_{n}(x) y^{n} \in R[y ; \alpha]$ with $p(y) q(y)=0$, where $f_{i}(x)$ and $g_{j}(x) \in R$ for all $0 \leq i \leq m$ and $0 \leq j \leq n$. Then we get $f_{i}(x) \alpha^{i}\left(g_{j}(x)\right)=0$ for all $i$ and $j$ by [8, Example 5], and so $f_{i}(x) R \alpha^{i}\left(g_{j}(x)\right)=0$. It is shown that for any $r \in R$ and nonnegative integer $t, p(y)\left(r y^{t}\right) q(y)=0 \Leftrightarrow p(y) R[y ; \alpha] q(y)=0$, concluding that $R[y ; \alpha]$ is semicommutative.

Corollary 2.11 ([11, Lemma $8(1)])$. Suppose that a ring $R$ is abelian. Then every idempotent of $R[x]$ is in $R$ and $R[x]$ is abelian.

Proposition 2.12. Let $R$ be a ring with an endomorphism $\alpha$. Assume that $a R \alpha(a)=0$ implies $a=0$ for any $a \in R$. The following are equivalent:

(1) $R$ is an $\alpha$-rigid ring (i.e., $R[x ; \alpha]$ is a reduced ring).

(2) $R[x ; \alpha]$ is a reversible ring.

(3) $R$ is an $\alpha$-shifting ring.

(4) $R$ is a right $\alpha$-shifting ring.

(5) $R[x ; \alpha]$ is a semicommutative ring.

(6) $R$ is a semicommutative ring.

Proof. Note that $R$ is $\alpha$-rigid if and only if $R[x ; \alpha]$ is reduced by [8, Proposition 3]. $(1) \Rightarrow(2)$ and $(3) \Rightarrow(4)$ are obvious, $(2) \Rightarrow(3)$ and $(4) \Rightarrow(1)$ follow from Theorem $2.9(3)$ and Theorem 2.6(5), respectively. $(2) \Rightarrow(5) \Rightarrow(6)$ are obvious. $(6) \Rightarrow(1)$ : Assume (6). For $a \in R$, if $a \alpha(a)=0$, then $a R \alpha(a)=0$ since $R$ is semicommutative, and hence $a=0$ by assumption. The proof is completed.

Corollary 2.13. Let $R[x]$ denote the polynomial ring with an indeterminate $x$ over a ring $R$. Assume that $R$ is a semiprime ring. Then $R$ is a reduced ring (i.e., $R[x]$ is a reduced ring) if and only if $R[x]$ is a reversible ring if and only if $R$ is a reversible ring if and only if $R[x]$ is a semicommutative ring if and only if $R$ is a semicommutative ring.

\section{Extensions of $\alpha$-shifting rings}

For $n \geq 2$, let $\operatorname{Mat}_{n}(R)$ (resp., $U_{n}(R)$ ) denote the $n \times n$ full matrix ring (resp., upper triangular matrix ring) over a ring $R$. For an endomorphism $\alpha$ 
of $R$, the map $\bar{\alpha}: \operatorname{Mat}_{n}(R) \rightarrow \operatorname{Mat}_{n}(R)$ defined by $\bar{\alpha}\left(\left(a_{i j}\right)\right)=\left(\alpha\left(a_{i j}\right)\right)$ is an endomorphism of $\operatorname{Mat}_{n}(R)$. Notice that the extended map $\bar{\alpha}$ of any subring $S$ with $\alpha(S) \subseteq S$ of $\operatorname{Mat}_{n}(R)$ for $n \geq 2$ is similarly defined component-wise.

Use $e_{i j}$ for the matrix with $(i, j)$-entry 1 and elsewhere 0 .

Proposition 3.1. Let $R$ be a ring with an endomorphism $\alpha$. For $n \geq 2$ and nonzero $\alpha$, if $\operatorname{Mat}_{n}(R)\left(U_{n}(R)\right)$ is right (resp., left) $\bar{\alpha}$-shifting, then $R$ is right (resp., left) $\alpha$-shifting.

Proof. Assume that $\operatorname{Mat}_{n}(R)$ for $n \geq 2$ is right $\bar{\alpha}$-shifting. Let $a \alpha(b)=0$ for $a, b \in R$. For $A=a \sum_{i=1}^{n} e_{i i}, \bar{B}=b \sum_{i=1}^{n} e_{i i} \in \operatorname{Mat}_{n}(R), A \bar{\alpha}(B)=$ $\left(a \sum_{i=1}^{n} e_{i i}\right)\left(\alpha(b) \sum_{i=1}^{n} e_{i i}\right)=0$, and so $B \bar{\alpha}(A)=0$ by assumption. Thus, $b \alpha(a)=0$, showing that $R$ is right $\alpha$-shifting. The proof for $U_{n}(R)$ is similar.

The converse of Proposition 3.1 does not hold for any nonzero endomorphism $\alpha$, by the following example.

Example 3.2. Let $\alpha$ be a nonzero endomorphism of a ring $R$. For $A=$ $e_{n n}, B=e_{1 n} \in \operatorname{Mat}_{n}(R), A \bar{\alpha}(B)=0$, but $B \bar{\alpha}(A)=\alpha(1) e_{1 n} \neq 0$ for any $n \geq 2$. Similarly, for any $n \geq 2$ and nonzero $\alpha, U_{n}(R)$ is not right $\bar{\alpha}$-shifting, either.

Given a ring $R$ and an $(R, R)$-bimodule $M$, the trivial extension of $R$ by $M$ is the $\operatorname{ring} T(R, M)=R \oplus M$ with the usual addition and the following multiplication: $\left(r_{1}, m_{1}\right)\left(r_{2}, m_{2}\right)=\left(r_{1} r_{2}, r_{1} m_{2}+m_{1} r_{2}\right)$. This is isomorphic to the ring of all matrices $\left(\begin{array}{cc}r & m \\ 0 & r\end{array}\right)$, where $r \in R$ and $m \in M$ and the usual matrix operations are used. The trivial extension $T(R, R)$ of a right $\alpha$-shifting ring $R$ need not to be so, in general [12, Example 1.7]. However, we have the following.

Proposition 3.3. Let $R$ be a reduced ring with an endomorphism $\alpha$. A ring $R$ is $\alpha$-shifting if and only if $T(R, R)$ is $\bar{\alpha}$-shifting.

Proof. It is enough to show the necessity for the right case. Suppose that $R$ is a right $\alpha$-shifting ring. Let $A=\left(\begin{array}{ll}a & b \\ 0 & a\end{array}\right), B=\left(\begin{array}{ll}c & d \\ 0 & c\end{array}\right) \in T(R, R)$ with $A \bar{\alpha}(B)=0$. Then $a \alpha(c)=0$ and $a \alpha(d)+b \alpha(c)=0$. From $a \alpha(c)=0$, we have $a R \alpha(c)=0$ and $c \alpha(a)=0$ since $R$ is reduced right $\alpha$-shifting. Hence, $0=a(a \alpha(d)+$ $b \alpha(c))=a^{2} \alpha(d)=a \alpha(d) a$ yields $a \alpha(d)=0$ and $b \alpha(c)=0$. Since $R$ is right $\alpha$ shifting, $d \alpha(a)=0$ and $c \alpha(b)=0$, proving that $T(R, R)$ is right $\bar{\alpha}$-shifting.

Corollary 3.4 ([12, Proposition 1.6]). Let $R$ be a reduced ring. Then $T(R, R)$ is a reversible ring.

For a ring $R$ and $n \geq 3$, let

$$
S_{n}(R)=\left\{\left(\begin{array}{ccccc}
a & a_{12} & a_{13} & \cdots & a_{1 n} \\
0 & a & a_{23} & \cdots & a_{2 n} \\
0 & 0 & a & \cdots & a_{3 n} \\
\vdots & \vdots & \vdots & \ddots & \vdots \\
0 & 0 & 0 & \cdots & a
\end{array}\right) \mid a, a_{i j} \in R\right\} .
$$


By the same arguments as in the proof of Proposition 3.1, we see that $R$ is right (resp., left) $\alpha$-shifting if $S_{n}(R)$ for $n \geq 2$ is right (resp., left) $\bar{\alpha}$-shifting. It is natural to ask whether $S_{n}(R)$ for $n \geq 3$ is $\bar{\alpha}$-shifting, when $R$ is a reduced ring. However, the answer is negative by the similar method to Example 3.2: Let $R$ be any ring and $\alpha$ a nonzero endomorphism of $R$. For $S_{n}(R)$ with $n \geq 3$, let $A=e_{23}$ and $B=e_{12} \in S_{n}(R)$. Then $A \bar{\alpha}(B)=0$, but $B \bar{\alpha}(A) \neq 0$, entailing that $S_{n}(R)$ is not right $\bar{\alpha}$-shifting for $n \geq 3$.

Proposition 3.5. Let $\alpha$ be an endomorphism of a ring $R, S$ a ring and $\sigma$ : $R \rightarrow S$ a ring isomorphism. Then $R$ is a right (resp., left) $\alpha$-shifting ring if and only if $S$ is a right (resp., left) $\sigma \alpha \sigma^{-1}$-shifting ring.

Proof. For $a, b \in R$, let $a^{\prime}=\sigma(a)$ and $b^{\prime}=\sigma(b)$. Then $a^{\prime} \sigma \alpha \sigma^{-1}\left(b^{\prime}\right)=0 \Leftrightarrow$ $\sigma(a \alpha(b))=0 \Leftrightarrow a \alpha(b)=0$. This fact follows that $R$ is right $\alpha$-shifting if and only if $S$ is right $\sigma \alpha \sigma^{-1}$-shifting.

Now, we consider the Jordan extension and Dorroh extension of $\alpha$-shifting rings. Recall that for a monomorphism $\alpha$ of a ring $R$, an over-ring $A$ of $R$ is a Jordan extension of $R$ if $\alpha$ can be extended to an automorphism of $A$ and $A=\cup_{n=0}^{\infty} \alpha^{-n}(R)$. Jordan [10] showed, with the use of left localization of the skew polynomial ring $R[x ; \alpha]$ with respect to the set of powers of $x$, that for any pair $(R, \alpha)$, such an extension $A$ always exists. On the other hand, for an algebra $R$ over a nonzero commutative ring $S$, the Dorroh extension of $R$ by $S$ is the ring $D=R \times S$ with operations $\left(r_{1}, s_{1}\right)+\left(r_{2}, s_{2}\right)=\left(r_{1}+r_{2}, s_{1}+s_{2}\right)$ and $\left(r_{1}, s_{1}\right)\left(r_{2}, s_{2}\right)=\left(r_{1} r_{2}+s_{1} r_{2}+s_{2} r_{1}, s_{1} s_{2}\right)$, where $r_{i} \in R$ and $s_{i} \in S$. For an $S$-endomorphism $\alpha$ of $R$ and the Dorroh extension $D$ of $R$ by $S$, the nonzero map $\bar{\alpha}: D \rightarrow D$ defined by $\bar{\alpha}(r, s)=(\alpha(r), s)$ is an $S$-algebra homomorphism.

Theorem 3.6. For an endomorphism $\alpha$ of a ring $R$, we have the following.

(1) Let $A$ be the corresponding Jordan extension of $R$ and $\alpha$ be a monomorphism of $R$. Then $R$ is a right (resp., left) $\alpha$-shifting ring if and only if $A$ is a right (resp., left) $\alpha$-shifting ring.

(2) Let $S$ be a domain and $\alpha$ be a monomorphism of $R$. Then $R$ is a right (resp., left) $\alpha$-shifting ring if and only if the Dorroh extension $D$ of $R$ by $S$ is right (resp., left) $\bar{\alpha}$-shifting.

Proof. If $R$ is a right $\alpha$-shifting ring, then so is any subring $S$ with $\alpha(S) \subseteq S$. Hence, it is enough to show the necessity.

(1) Suppose that $R$ is right $\alpha$-shifting and $a \alpha(b)=0$ for $a, b \in A$. By the definition of $A$, there exists $k \geq 0$ such that $\alpha^{k}(a), \alpha^{k}(b) \in R$. Then $\alpha^{k}(a) \alpha\left(\alpha^{k}(b)\right)=\alpha^{k}(a \alpha(b))=\alpha^{k}(0)=0 \Rightarrow \alpha^{k}(b) \alpha\left(\alpha^{k}(a)\right)=0 \Rightarrow \alpha^{k}(b \alpha(a))=$ 0 . Hence, $b \alpha(a)=0$ since $\alpha$ is a monomorphism. Therefore $A$ is right $\alpha$ shifting.

(2) Suppose that $R$ is a right $\alpha$-shifting ring. Recall that $\alpha$ is a monomorphism if and only if $\alpha(1)=1$ for the identity 1 of $R$ by Theorem 2.6(3). Let $\left(r_{1}, s_{1}\right),\left(r_{2}, s_{2}\right) \in D$ with $\left(r_{1}, s_{1}\right) \bar{\alpha}\left(r_{2}, s_{2}\right)=0$. Then $r_{1} \alpha\left(r_{2}\right)+s_{1} \alpha\left(r_{2}\right)+s_{2} r_{1}=$ 
0 and $s_{1} s_{2}=0$. Since $S$ is a domain, $s_{1}=0$ or $s_{2}=0$. If $s_{1}=0$, then $0=r_{1} \alpha\left(r_{2}\right)+s_{2} r_{1}$ and so $0=r_{1}\left(\alpha\left(r_{2}\right)+s_{2}\right)=r_{1} \alpha\left(r_{2}+1 \cdot s_{2}\right) \Rightarrow$ $\left(r_{2}+s_{2}\right) \alpha\left(r_{1}\right)=0 \Rightarrow\left(r_{2}, s_{2}\right) \bar{\alpha}\left(r_{1}, s_{1}\right)=0$. Similarly, let $s_{2}=0$. Then $\left(r_{1}+s_{1}\right) \alpha\left(r_{2}\right)=0 \Rightarrow r_{2} \alpha\left(r_{1}+s_{1}\right)=0 \Rightarrow\left(r_{2}, s_{2}\right) \bar{\alpha}\left(r_{1}, s_{1}\right)=0$, and thus the Dorroh extension $D$ is right $\bar{\alpha}$-shifting.

The condition " $\alpha$ is a monomorphism of $R$ " in Theorem 3.6(2) is not superfluous by the next example.

Example 3.7. For the ring $\mathbb{Z}_{2}$ of integers modulo 2 , let $R=\mathbb{Z}_{2} \oplus \mathbb{Z}_{2}$ and $\alpha: R \rightarrow R$ be defined by $\alpha((a, b))=(0, b)$. Then $\alpha$ is not a monomorphism of $R$. Note that $R$ is right $\alpha$-shifting: Indeed, let $A \alpha(B)=0$ for $A=(a, b), B=$ $(c, d) \in R$. Then $b=0$ or $d=0$. If $b=0$, then $B \alpha(A)=(c, d)(0,0)=0$. If $d=0$, then $B \alpha(A)=(c, 0)(0, b)=0$. Consequently, $R$ is right $\alpha$-shifting. Now, consider the Dorroh extension $D$ of $R$ by the ring of integers $\mathbb{Z}$. Then $((1,0),-1) \bar{\alpha}((1,0), 0)=0$, but $((1,0), 0) \bar{\alpha}((1,0),-1)=(-(1,0), 0) \neq 0$ in $D$, showing that the Dorroh extension $D$ of $R$ by $\mathbb{Z}$ is not right $\bar{\alpha}$-shifting.

Corollary 3.8 ([12, Proposition $1.14(2)])$. Let $R$ be an algebra over a commutative ring $S$, and $D$ be the Dorroh extension of $R$ by $S$. If $R$ is reversible and $S$ is a domain, then $D$ is also reversible.

Rege and Chhawchharia called a ring $R$ Armendariz [20, Definition 1.1] if whenever the product of any two polynomials in $R[x]$ over $R$ is zero, then so is the product of any pair of coefficients from the two polynomials. Any reduced ring is Armendariz by [1, Lemma 1], but the converse is not true in general.

For an endomorphism $\alpha$ of a ring $R$, the map $\bar{\alpha}: R[x] \rightarrow R[x]$ defined by $\bar{\alpha}\left(\sum_{i=0}^{m} a_{i} x^{i}\right)=\sum_{i=0}^{m} \alpha\left(a_{i}\right) x^{i}$ is an endomorphism of the polynomial ring $R[x]$ and clearly this map extends $\alpha$. The ring of Laurent polynomials in $x$, coefficients in a ring $R$, consists of all formal sums $\sum_{i=k}^{n} m_{i} x^{i}$ with obvious addition and multiplication, where $m_{i} \in R$ and $k, n$ are (possibly negative) integers; denote it by $R\left[x ; x^{-1}\right]$. The map $\bar{\alpha}: R\left[x, x^{-1}\right] \rightarrow R\left[x, x^{-1}\right]$ defined by $\bar{\alpha}\left(\sum_{i=k}^{n} a_{i} x^{i}\right)=\sum_{i=k}^{n} \alpha\left(a_{i}\right) x^{i}$ extends $\alpha$ and is also an endomorphism of $R\left[x, x^{-1}\right]$.

Proposition 3.9. Let $R$ be a ring with an endomorphism $\alpha$. Then the following are equivalent:

(1) $R[x]$ is a right (resp., left) $\bar{\alpha}$-shifting ring.

(2) $R\left[x ; x^{-1}\right]$ is a right (resp., left) $\bar{\alpha}$-shifting ring.

If $R$ is an Armendariz ring, then we add in

(3) $R$ is a right (resp., left) $\alpha$-shifting ring.

Proof. $(2) \Rightarrow(1) \Rightarrow(3)$ are clear as subrings. $(1) \Rightarrow(2)$ Suppose that $R[x]$ is right $\bar{\alpha}$-shifting. Let $f(x), g(x) \in R\left[x ; x^{-1}\right]$ with $f(x) \bar{\alpha}(g(x))=0$. Then there exists a positive integer $n$ such that $f_{1}(x)=f(x) x^{n}, g_{1}(x)=g(x) x^{n} \in R[x]$ with $f_{1}(x) \bar{\alpha}\left(g_{1}(x)\right)=0$. Since $R[x]$ is right $\bar{\alpha}$-shifting, we obtain $g_{1}(x) \bar{\alpha}\left(f_{1}(x)\right)=$ 
0. Hence $g(x) \bar{\alpha}(f(x))=x^{-2 n} g_{1}(x) \bar{\alpha}\left(f_{1}(x)\right)=0$. Thus $R\left[x ; x^{-1}\right]$ is right $\bar{\alpha}$ shifting. $(3) \Rightarrow(1)$ Assume that $R$ is right $\alpha$-shifting. Let $f(x)=a_{0}+a_{1} x+$ $\cdots+a_{n} x^{n}, g(x)=b_{0}+b_{1} x+\cdots+b_{m} x^{m} \in R[x]$ with $f(x) \bar{\alpha}(g(x))=0$. Since $R$ is Armendariz and right $\alpha$-shifting, we get $a_{i} \alpha\left(b_{j}\right)=0$ and so $b_{j} \alpha\left(a_{i}\right)=0$ for all $i, j$, proving that $R[x]$ is right $\bar{\alpha}$-shifting.

The condition " $R$ is an Armendariz ring" in Proposition 3.9 is not superfluous by the next example.

Example 3.10. The $2 \times 2$ upper triangular matrix ring over any ring $R$ is not Armendariz by [11, Example 1]. Hence, the $\operatorname{ring} R=\left\{\left(\begin{array}{ll}a & b \\ 0 & c\end{array}\right) \mid a, b, c \in \mathbb{Z}\right\}$ and $\alpha$ defined by $\alpha\left(\left(\begin{array}{ll}a & b \\ 0 & c\end{array}\right)\right)=\left(\begin{array}{ll}a & 0 \\ 0 & 0\end{array}\right)$, in Example 2.5(1), is right $\alpha$-shifting, but not Armendariz. For $f(x)=\left(\begin{array}{ll}0 & 1 \\ 0 & 0\end{array}\right)+\left(\begin{array}{ll}0 & 1 \\ 0 & 1\end{array}\right) x, g(x)=\left(\begin{array}{ll}1 & 0 \\ 0 & 0\end{array}\right)+\left(\begin{array}{ll}1 & 1 \\ 0 & 0\end{array}\right) x \in R[x]$, we have $f(x) \bar{\alpha}(g(x))=0$, but $g(x) \bar{\alpha}(f(x))=\left(\begin{array}{ll}0 & 1 \\ 0 & 0\end{array}\right)+\left(\begin{array}{ll}0 & 1 \\ 0 & 0\end{array}\right) x \neq 0$, showing that $R[x]$ is not right $\bar{\alpha}$-shifting.

Corollary 3.11 ([12, Proposition 2.4]). Let $R$ be an Armendariz ring. The following are equivalent:

(1) $R$ is reversible.

(2) $R[x]$ is reversible.

(3) $R\left[x ; x^{-1}\right]$ is reversible.

For an ideal $I$ and an endomorphism $\alpha$ of a ring $R, I$ is called an $\alpha$-ideal if $\alpha(I) \subseteq I$; for an $\alpha$-ideal $I$ the map $\bar{\alpha}: R / I \rightarrow R / I$ defined by $\bar{\alpha}(a+I)=$ $\alpha(a)+I$ is an endomorphism of a factor $\operatorname{ring} R / I$. The homomorphic image of a reversible ring may not necessarily be reversible in general. The next example shows that there exists a $\operatorname{ring} R$ with an endomorphism $\alpha$ which is not right $\alpha$-shifting, even though $R / I$ is right $\bar{\alpha}$-shifting for a nonzero proper $\alpha$-ideal $I$ of $R$.

Example 3.12. Let $R=\left(\begin{array}{cc}F & F \\ 0 & F\end{array}\right)$ and an endomorphism $\alpha$ be defined by $\alpha\left(\left(\begin{array}{ll}a & b \\ 0 & c\end{array}\right)\right)$ $=\left(\begin{array}{cc}a & -b \\ 0 & c\end{array}\right)$, where $F$ is a field. For $A=\left(\begin{array}{ll}0 & 1 \\ 0 & 0\end{array}\right), B=\left(\begin{array}{ll}1 & 1 \\ 0 & 0\end{array}\right) \in R$, we have $A \alpha(B)=0$ but $B \alpha(A) \neq 0$, entailing that $R$ is not right $\alpha$-shifting. For an ideal $I=\left(\begin{array}{ll}0 & F \\ 0 & 0\end{array}\right)$ of $R$, the factor $\operatorname{ring} R / I=\left\{\left(\begin{array}{ll}a & 0 \\ 0 & c\end{array}\right)+I \mid a, c \in F\right\}$ is reduced and so right $\bar{\alpha}$-shifting where $\bar{\alpha}$ is an identity map on $R / I$. Note that $I$ is not an $\alpha$-rigid ring (without identity).

However, we have the following.

Theorem 3.13. Let $R$ be a ring with an endomorphism $\alpha$.

(1) For an $\alpha$-ideal $I$ of $R$, let $R / I$ be a right (resp., left) $\bar{\alpha}$-shifting ring and $I$ be an $\alpha$-rigid ring without identity. Then $R$ is a right (resp., left) $\alpha$-shifting ring.

(2) Let $R$ be a reduced ring and $n$ be any positive integer. $R$ is a right (resp., left) $\alpha$-shifting ring if and only if $R[x] /\left\langle x^{n}\right\rangle$ is a right (resp., left) $\bar{\alpha}$-shifting ring, where $\left\langle x^{n}\right\rangle$ is the ideal generated by $x^{n}$. 
Proof. (1) Let $a \alpha(b)=0$ for $a, b \in R$. Then $b \alpha(a) \in I$ since $R / I$ is right $\bar{\alpha}$-shifting. Then $a \alpha(b \alpha(a))=0 \Rightarrow b \alpha(a) a \alpha(b \alpha(a) a)=0 \Rightarrow b \alpha(a) a=0 \Rightarrow$ $a b \alpha(a)=0 \Rightarrow b \alpha(a) \alpha(b \alpha(a))=0 \Rightarrow b \alpha(a)=0$, since $b \alpha(a) \in I$ and $I$ is $\alpha$-rigid (and so reduced). Thus $R$ is right $\alpha$-shifting.

(2) It suffices to show that the necessity. Assume that $R$ is a right $\alpha$ shifting ring. Let $S=R[x] /\left\langle x^{n}\right\rangle$. If $n=1$, then $S \cong R$. If $n=2$, then $S \cong T(R, R)$ is $\bar{\alpha}$-shifting by Proposition 3.3. Now, we assume that $n \geq 3$. Let $f=a_{0}+a_{1} t+\cdots+a_{n-1} t^{n-1}, g=b_{0}+b_{1} t+\cdots+b_{n-1} t^{n-1} \in S$ with $f \bar{\alpha}(g)=0$, where $t=x+\left\langle x^{n}\right\rangle$. We claim that $b_{j} \alpha\left(a_{i}\right)=0$ for all $i$ and $j$. We proceed by induction on $i+j$. Note that if $i+j \geq n$ for all $i$ and $j$, then $a_{i} \alpha\left(b_{j}\right) t^{i+j}=0$. Hence we set $i+j \leq n-1$. From $f \bar{\alpha}(g)=0$, we get $a_{0} \alpha\left(b_{0}\right)=0$ and so $b_{0} \alpha\left(a_{0}\right)=0$, proving for $i+j=0$. Now assume that our claim is true for $i+j \leq k-1$. For $i+j=k$, we have

$$
a_{0} \alpha\left(b_{k}\right)+a_{1} \alpha\left(b_{k-1}\right)+a_{2} \alpha\left(b_{k-2}\right)+\cdots+a_{k-1} \alpha\left(b_{1}\right)+a_{k} \alpha\left(b_{0}\right)=0 .
$$

Recall that $R$ is a reduced ring if and only if $a b^{2}=0$ implies $a b=0$ for any $a, b \in R$, and every reduced ring is reversible. We freely use these facts in the following. Multiplying Eq. (2) by $\alpha\left(\alpha\left(a_{0}\right)\right)$ on the right hand-side, we get $a_{0} \alpha\left(b_{k} \alpha\left(a_{0}\right)\right)=0$ by induction hypothesis and assumption. Hence, $b_{k}\left(\alpha\left(a_{0}\right)\right)^{2}=$ 0 and so $b_{k} \alpha\left(a_{0}\right)=0$ and $a_{0} \alpha\left(b_{k}\right)=0$. Eq.(2) becomes

$$
a_{1} \alpha\left(b_{k-1}\right)+a_{2} \alpha\left(b_{k-2}\right)+\cdots+a_{k-1} \alpha\left(b_{1}\right)+a_{k} \alpha\left(b_{0}\right)=0 .
$$

Multiplying Eq.(3) by $\alpha\left(\alpha\left(a_{1}\right)\right)$ on the right hand-side, by a similar way we get $b_{k-1} \alpha\left(a_{1}\right)=0$ and hence $a_{1} \alpha\left(b_{k-1}\right)=0$, and Eq.(3) becomes

$$
a_{2} \alpha\left(b_{k-2}\right)+\cdots+a_{k-1} \alpha\left(b_{1}\right)+a_{k} \alpha\left(b_{0}\right)=0 .
$$

Multiplying Eq.(4) by $\alpha\left(\alpha\left(a_{2}\right)\right)$ on the right hand-side, we get $b_{k-2} \alpha\left(a_{2}\right)=$ 0 and so $a_{2} \alpha\left(b_{k-2}\right)=0$ by the same arguments as above. Continuing this procedure yields $b_{j} \alpha\left(a_{i}\right)=0$ for $i+j=k$, and consequently $b_{j} \alpha\left(a_{i}\right)=0$ for all $i$ and $j$. Thus $g \bar{\alpha}(f)=0$, proving that $S$ is right $\bar{\alpha}$-shifting.

For a ring $R$ and $n \geq 2$, let

$$
V_{n}(R)=\left\{\left(\begin{array}{cccccc}
a_{1} & a_{2} & a_{3} & a_{4} & \cdots & a_{n} \\
0 & a_{1} & a_{2} & a_{3} & \cdots & a_{n-1} \\
0 & 0 & a_{1} & a_{2} & \cdots & a_{n-2} \\
\vdots & \vdots & \vdots & \vdots & \cdots & \vdots \\
0 & 0 & 0 & 0 & \cdots & a_{2} \\
0 & 0 & 0 & 0 & \cdots & a_{1}
\end{array}\right) \mid a_{1}, a_{2}, \ldots, a_{n} \in R\right\} .
$$

The next corollary which follows directly from Theorem 3.13(2) can be compared with Example 3.2.

Corollary 3.14. Assume that $R$ is a reduced ring with an endomorphism $\alpha$. Then $R$ is an $\alpha$-shifting ring if and only if $V_{n}(R)$ is an $\bar{\alpha}$-shifting ring for any $n \geq 2$. 
Proof. Note that $V_{n}(R) \cong R[x] /\left\langle x^{n}\right\rangle$ by $[15]$.

Corollary 3.15. (1) ([12, Proposition 1.12]) Suppose that $R / I$ is a reversible ring for some ideal $I$ of a ring $R$. If $I$ is reduced, then $R$ is reversible.

(2) ([12, Theorem 2.5]) If $R$ is a reduced ring, then $R[x] /\left\langle x^{n}\right\rangle\left(\cong V_{n}(R)\right)$ is a reversible ring for any positive integer $n$.

Recall that an element $u$ of a ring $R$ is right regular if $u r=0$ implies $r=0$ for $r \in R$. Similarly, left regular is defined, and regular means if it is both left and right regular (and hence not a zero divisor). Let $\Delta$ be a multiplicatively closed subset of $R$ consisting of central regular elements. For an automorphism $\alpha$ of $R$ with $\alpha(\Delta) \subseteq \Delta$, the induced map $\bar{\alpha}: \Delta^{-1} R \rightarrow \Delta^{-1} R$ defined by $\bar{\alpha}\left(u^{-1} a\right)=\alpha(u)^{-1} \alpha(a)$ is also an automorphism.

Proposition 3.16. Let $R$ be a ring with an automorphism $\alpha$. Assume that there exists a multiplicatively closed subset $\Delta$ of $R$ consisting of central regular elements. Then $R$ is a right (resp., left) $\alpha$-shifting ring if and only if $\Delta^{-1} R$ is a right (resp., left) $\bar{\alpha}$-shifting ring.

Proof. It is enough to show the necessity. Assume that $R$ is right $\alpha$-shifting. Let $A \bar{\alpha}(B)=0$ for $A=u^{-1} a, B=v^{-1} b$, where $a, b, u, v \in R$ with $u, v$ regular. Since $\Delta$ is contained in the center of $R$, we have $0=A \bar{\alpha}(B)=$ $u^{-1} a \bar{\alpha}\left(v^{-1} b\right)=(u \alpha(v))^{-1} a \alpha(b)$ and so $a \alpha(b)=0$. By assumption, $b \alpha(a)=0$. Then $0=(v \alpha(u))^{-1} b \alpha(a)=v^{-1} b \bar{\alpha}\left(u^{-1} a\right)=B \bar{\alpha}(A)$, proving that $\Delta^{-1} R$ is right $\bar{\alpha}$-shifting.

The following corollary is compared with Proposition 3.9.

Corollary 3.17. Let $R$ be a ring with an automorphism $\alpha$. Then $R[x]$ is a right (resp., left) $\bar{\alpha}$-shifting ring if and only if $R\left[x ; x^{-1}\right]$ is a right (resp., left) $\bar{\alpha}$-shifting ring.

Proof. It follows directly from Proposition 3.16. For, let $\Delta=\left\{1, x, x^{2}, \ldots\right\}$, then clearly $\Delta$ is a multiplicatively closed subset of $R[x]$ and $R\left[x ; x^{-1}\right]=$ $\Delta^{-1} R[x]$.

A ring $R$ is called right (resp., left) Ore if given $a, b \in R$ with $b$ regular there exist $a_{1}, b_{1} \in R$ with $b_{1}$ regular such that $a b_{1}=b a_{1}$ (resp., $b_{1} a=a_{1} b$ ). It is a well-known fact that $R$ is a right (resp., left) Ore ring if and only if the classical right (resp., left) quotient ring of $R$ exists.

Suppose that the classical right quotient ring $Q(R)$ of $R$ exists. Then for an automorphism $\alpha$ of $R$ and any $a b^{-1} \in Q(R)$ where $a, b \in R$ with $b$ regular, the induced map $\bar{\alpha}: Q(R) \rightarrow Q(R)$ defined by $\bar{\alpha}\left(a b^{-1}\right)=\alpha(a) \alpha(b)^{-1}$ is also an automorphism.

Theorem 3.18. Let $R$ be a right Ore ring, and $\alpha$ an automorphism of $R$, and $Q(R)$ the classical right quotient ring of $R$. Then $R$ is a right (resp., left) $\alpha$-shifting ring if and only if $Q(R)$ is a right (resp., left) $\bar{\alpha}$-shifting ring. 
Proof. It suffices to establish the necessity. We freely use the result of $[16$, Proposition 2.1.16]. Assume that $R$ is right $\alpha$-shifting. Let $A=a b^{-1}$ and $B=c d^{-1}$ with $A \bar{\alpha}(B)=0$, where $a, b, c, d \in R$ with $b, d$ regular. There exist $b_{1}, c_{1} \in R$ with $b_{1}$ regular such that $b c_{1}=\alpha(c) b_{1}$ and $c_{1} b_{1}^{-1}=b^{-1} \alpha(c)$. Hence, $0=A \bar{\alpha}(B)=a b^{-1} \alpha(c) \alpha(d)^{-1}=a c_{1} b_{1}^{-1} \alpha(d)^{-1}$. Then $0=a c_{1}=a \alpha\left(c^{\prime}\right)$, putting $c_{1}=\alpha\left(c^{\prime}\right)$ for some $c^{\prime} \in R$. Since $R$ is right $\alpha$-shifting, $c^{\prime} \alpha(a)=0 \Rightarrow$ $c^{\prime} \alpha(a) \alpha(b)=0 \Rightarrow a b c_{1}=0 \Rightarrow a \alpha(c) b_{1}=0 \Rightarrow a \alpha(c)=0$ and $c \alpha(a)=0$. Now, for $a, d \in R$ there exist $a_{1}, d_{1} \in R$ with $d_{1}$ regular such that $d a_{1}=$ $\alpha(a) d_{1}$ and $a_{1} d_{1}^{-1}=d^{-1} \alpha(a)$. From $c \alpha(a)=0$, we get $\alpha(c \alpha(a)) \alpha\left(d_{1}\right)=$ $0 \Rightarrow \alpha(a) d_{1} \alpha^{2}(c)=0 \Rightarrow d a_{1} \alpha^{2}(c)=0 \Rightarrow a_{1} \alpha^{2}(c)=0 \Rightarrow c a_{1}=0$. Thus $B \bar{\alpha}(A)=c d^{-1} \alpha(a) \alpha(b)^{-1}=c a_{1} d_{1}^{-1} \alpha(b)^{-1}=0$, concluding that $Q(R)$ is right $\bar{\alpha}$-shifting.

Corollary 3.19 ([12, Theorem 2.6]). Let $R$ be a right Ore ring and $Q(R)$ be the classical right quotient ring of $R$. Then $R$ is reversible if and only if $Q(R)$ is reversible.

Corollary 3.20. Let $R$ be a right Ore ring, and $\alpha$ an automorphism of $R$, and $Q(R)$ the classical right quotient ring of $R$. Assume that aR $\alpha(a)=0$ implies $a=0$ for any $a \in R$. The following are equivalent:

(1) $R$ is an $\alpha$-rigid ring .

(2) $R$ is an $\alpha$-shifting ring.

(3) $Q(R)$ is a $\bar{\alpha}$-rigid ring.

(4) $Q(R)$ is a $\bar{\alpha}$-shifting ring.

Proof. We claim that if $R$ satisfies that $a R \alpha(a)=0$ implies $a=0$ for any $a \in R$ then so is $Q(R)$. For, let $a b^{-1} Q(R) \bar{\alpha}\left(a b^{-1}\right)=0$. Then $0=a b^{-1} Q(R) \bar{\alpha}\left(a b^{-1}\right)=$ $a Q(R) \alpha(a) \alpha(b)^{-1}$, since $b^{-1} Q(R)=Q(R)$. This implies $a Q(R) \alpha(a)=0$, and so $a R \alpha(a)=0$. By assumption, we get $a=0$, completing the claim. Thus the proof is done by Proposition 2.12, Theorem 3.18 and the claim.

\section{A related topic}

A ring $R$ is called right (resp., left) McCoy [19] if whenever any nonzero polynomials $f(x), g(x) \in R[x]$ satisfy $f(x) g(x)=0$, then $f(x) c=0$ (resp., $c g(x)=0$ ) for some nonzero $c \in R$, and a ring $R$ is called McCoy if it is both left and right McCoy. Every Armendariz ring is McCoy but the converse does not hold by [20, Remark 4.3]. The Armendariz property and the McCoy condition of a ring were extended to the skew polynomial ring, respectively. A ring $R$ is called $\alpha$-skew Armendariz [8, Definition] if for $p(x)=a_{0}+a_{1} x+\cdots+a_{m} x^{m}$ and $q(x)=b_{0}+b_{1} x+\cdots+b_{n} x^{n}$ in $R[x ; \alpha], p(x) q(x)=0$ implies $a_{i} \alpha^{i}\left(b_{j}\right)=0$ for all $0 \leq i \leq m$ and $0 \leq j \leq n ; R$ is called an $\alpha$-skew McCoy ring [4, Definition 1] if for any nonzero skew polynomials $p(x)=\sum_{i=0}^{m} a_{i} x^{i}$ and $q(x)=$ $\sum_{j=0}^{n} b_{j} x^{j} \in R[x ; \alpha], p(x) q(x)=0$ implies $p(x) c=0$ for some nonzero $c \in R$. Any $\alpha$-rigid ring is $\alpha$-skew Armendariz, and any $\alpha$-skew Armendariz ring is $\alpha$-skew McCoy, but the converses do not hold in general. Notice that every 
reversible ring is McCoy by [19, Theorem 2]; every reversible and $\alpha$-reversible ring with a monomorphism is $\alpha$-skew McCoy by [4, Theorem 9(1)], but there exists a commutative reduced $\alpha$-shifting ring with an automorphism $\alpha$ which is not an $\alpha$-skew McCoy ring: For example, the commutative reduced $\alpha$-shifting ring $R=\mathbb{Z}_{2} \oplus \mathbb{Z}_{2}$ with an automorphism $\alpha$ defined by $\alpha((a, b))=(b, a)$ in Example 1.1(1) is not $\alpha$-skew McCoy by [4, Example 4]. However, right $\alpha$ shifting rings satisfying that $a R \alpha(a)=0$ for $a \in R$ implies $a=0$ (i.e., $\alpha$-rigid rings) is $\alpha$-skew Armendariz by [8, Corollary 4], and so $\alpha$-skew McCoy.

Finally, we give a positive answer to a question posed in [4] about the relationship between skew McCoy condition of rings and their classical right quotient rings.

Proposition 4.1. Let $R$ be a right Ore ring, and $\alpha$ an automorphism of $R$, and $Q(R)$ the classical right quotient ring of $R$. Then

(1) For each $q(x) \in Q(R)[x ; \bar{\alpha}]$, there exists a regular element $v \in R$ and $g(x) \in R[x ; \alpha]$ such that $q(x)=g(x) v^{-1}$.

(2) $A$ ring $R$ is $\alpha$-skew McCoy if and only if $Q(R)$ is an $\bar{\alpha}$-skew McCoy ring.

Proof. (1) It follows from the proof of [18, Theorem 4.7].

(2) We freely use the result of [16, Proposition 2.1.16]. Suppose that $R$ is $\alpha$-skew McCoy. Let $F(x)=\sum_{i=0}^{m} A_{i} x^{i}$ and $G(x)$ be nonzero polynomials in $Q(R)[x ; \bar{\alpha}]$ such that $F(x) G(x)=0$, where $A_{i}=a_{i} u^{-1}$ with $a_{i} \in R$ and regular $u \in R$. For each $i$, there exist $a^{\prime}{ }_{i} \in R$ and a regular $u^{\prime} \in R$ such that $u^{\prime-1} a^{\prime}{ }_{i}=a_{i} u^{-1}$. By (1), let $G(x)=g(x) v^{-1}$ for some regular element $v \in R$ and $0 \neq g(x) \in R[x ; \alpha]$. Then $0=F(x) G(x)=\left(u^{\prime-1} \sum_{i=0}^{m} a_{i}^{\prime} x^{i}\right) g(x) v^{-1}$ and so we get $\left(\sum_{i=0}^{m} a^{\prime}{ }_{i} x^{i}\right) g(x)=0$ in $R[x ; \alpha]$. Since $R$ is $\alpha$-skew McCoy, there exists $0 \neq c \in R$ such that $\left(\sum_{i=0}^{m} a^{\prime}{ }_{i} x^{i}\right) c=0$, and hence $F(x) c=0$. Therefore $Q(R)$ is a $\bar{\alpha}$-skew McCoy ring.

Conversely, assume that $Q(R)$ is $\bar{\alpha}$-skew McCoy. Let $f(x)=\sum_{i=0}^{m} a_{i} x^{i}$ and $g(x)=\sum_{j=0}^{n} b_{j} x^{j}$ be nonzero polynomials in $R[x ; \alpha]$ such that $f(x) g(x)=0$. Then there exists $0 \neq c u^{-1} \in Q(R)$ such that $f(x)\left(c u^{-1}\right)=0$ since $Q(R)$ is $\bar{\alpha}$-skew McCoy. Thus $f(x) c=0$ for $0 \neq c \in R$, and therefore $R$ is $\alpha$-skew $\mathrm{McCoy}$.

Acknowledgments. The authors would like to thank the referee for helpful comments and suggestions.

\section{References}

[1] E. P. Armendariz, A note on extensions of Baer and P.P.-rings, J. Austral. Math. Soc. 18 (1974), 470-473.

[2] D. D. Anderson and V. Camillo, Semigroups and rings whose zero products commute, Comm. Algebra 27 (1999), no. 6, 2847-2852.

[3] M. Başer, C. Y. Hong, and T. K. Kwak, On extended reversible rings, Algebra Colloq. 16 (2009), no. $1,37-48$. 
[4] M. Başer, T. K. Kwak, and Y. Lee, The McCoy condition on skew polynomial rings, Comm. Algebra 37 (2009), no. 11, 4026-4037.

[5] P. M. Cohn, Reversible rings, Bull. London Math. Soc. 31 (1999), no. 6, 641-648.

[6] J. M. Habeb, A note on zero commutative and duo rings, Math. J. Okayama Univ. 32 (1990), 73-76.

[7] C. Y. Hong, N. K. Kim, and T. K. Kwak, Ore extensions of Baer and p.p.-rings, J. Pure Appl. Algebra 151 (2000), no. 3, 215-226.

[8] _ On skew Armendariz rings, Comm. Algebra 31 (2003), no. 1, 103-122.

[9] C. Y. Hong, T. K. Kwak, and S. T. Rizvi, Extensions of generalized Armendariz rings, Algebra Colloq. 13 (2006), no. 2, 253-266.

[10] D. A. Jordan, Bijective extensions of injective ring endomorphisms, J. London Math. Soc. (2) 25 (1982), no. 3, 435-448.

[11] N. K. Kim and Y. Lee, Armendariz rings and reduced rings, J. Algebra 223 (2000), no. $2,477-488$

[12] _ Extensions of reversible rings, J. Pure Appl. Algebra 185 (2003), no. 1-3, 207223.

[13] J. Krempa, Some examples of reduced rings, Algebra Colloq. 3 (1996), no. 4, 289-300

[14] J. Lambek, On the representation of modules by sheaves of factor modules, Canad. Math. Bull. 14 (1971), 359-368.

[15] T. K. Lee and Y. Q. Zhou, Armendariz and reduced rings, Comm. Algebra 32 (2004), no. $6,2287-2299$

[16] J. C. McConnell and J. C. Robson, Noncommutative Noetherian Rings, John Wiley \& Sons Ltd., 1987.

[17] L. Motais de Narbonne, Anneaux semi-commutatifs et unisériels; anneaux dont les idéaux principaux sont idempotents, Proceedings of the 106th National Congress of Learned Societies (Perpignan, 1981), 71-73, Bib. Nat., Paris, 1982.

[18] A. R. Nasr-Isfahani and A. Moussavi, Ore extensions of skew Armendariz rings, Comm. Algebra 36 (2008), no. 2, 508-522.

[19] P. P. Nielsen, Semi-commutativity and the McCoy condition, J. Algebra 298 (2006), no. $1,134-141$.

[20] M. B. Rege and S. Chhawchharia, Armendariz rings, Proc. Japan Acad. Ser. A Math. Sci. 73 (1997), no. 1, 14-17.

[21] G. Y. Shin, Prime ideals and sheaf representation of a pseudo symmetric ring, Trans. Amer. Math. Soc. 184 (1973), 43-60.

MuhitTin BAŞER

Department of Mathematics

KOCATEPE UNIVERSITY

Afyonkarahisar 03200, Turkey

E-mail address: mbaser@aku.edu.tr

FATMA KAYNARCA

Department of Mathematics

KocATEPE UNIVERSITY

Afyonkarahisar 03200, Turkey

E-mail address: ferol@aku.edu.tr

Tai Keun Kwak

Department of Mathematics

DAEJin University

Pocheon 487-711, KoreA

E-mail address: tkkwak@daejin.ac.kr 